\title{
Erratum to: Candidates of trichocyst matrix proteins of the dinoflagellate Oxyrrhis marina
}

Erhard Rhiel $^{1} \cdot$ Lars Wöhlbrand $^{2} \cdot$ Ralf Rabus $^{2} \cdot$ Sonja Voget $^{3}$

Published online: 21 August 2017

(C) Springer-Verlag GmbH Austria 2017

\section{Erratum to: Protoplasma}

DOI 10.1007/s00709-017-1148-2

The published version contains mistake. Entries were incorrectly aligned in Table 1. Corrected table is shown below.

The online version of the original article can be found at http://dx.doi.org/ 10.1007/s00709-017-1148-2

Erhard Rhiel

erhard.rhiel@uni-oldenburg.de

1 Planktology, ICBM, Carl von Ossietzky University Oldenburg, P.O.B. 2503, 26129 Oldenburg, Germany

2 General and Molecular Microbiology, ICBM, Carl von Ossietzky University Oldenburg, P.O.B. 2503, 26129 Oldenburg, Germany

3 Stabsstelle Sicherheitswesen/Umweltschutz, Georg-August University Göttingen, Grisebachstr. 8, 37077 Göttingen, Germany 
Table 1 ClustalO sequence alignments of the relevant stretches of the deduced translation products of the ESTs of Oxyrrhis marina with the corresponding sequences of the mature TMPs of Paramecium tetraurelia (bold). Note that only the relevant parts of the deduced translation products of the ESTs of Oxyrrhis marina are shown. The entire amino acid sequences of the deduced translation products of the ESTs of Oxyrrhis marina and of the precursor and mature TMPs of Paramecium tetraurelia are compiled in Supplement II_Sequences

\begin{tabular}{|c|c|}
\hline T1-F1 & GILSELNSEIEKLIGEMEEELAGVHHEFNRRTDVHNREVARLEQEIQDKERELFNAHDFYDNVLI------PQGERFAAQLEQLQENIAHNRQTL \\
\hline CAMPEP 0190324578 & SFLEHTKNPVRKVVDLLQKLGKEIEDETSKAKKMYNKYMCYCDTNTTÁSLEKDIEEEKEHISELEANMKELSGQNAQLEAẼIADAOEESLEANKKTI \\
\hline CAMPEP-0190375660 & AVAAEGSSPVAKVVQLLSSLSAKVTAEGEREQQQYEKYVQWCEDNAKEKQHELKNSADTKENLEARVAKLQAS ISTTESHIEELSSS ISRNEQDL \\
\hline & 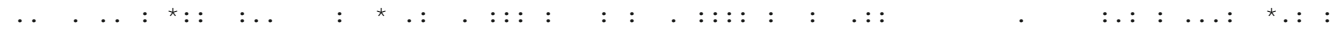 \\
\hline T1-F1 & EQATVQRANDHETFESEVVEHNDAISAIDECLQLLSTIAAPSLAE \\
\hline CAMPEP 0190324578 & AEATVMRNNEAAQYASESGELKDS IAALAKAIPALKKGMENGSFL \\
\hline CAMPEP_0190375660 & 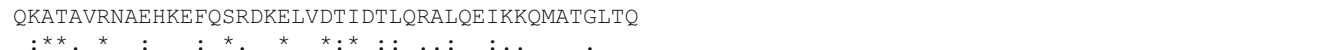 \\
\hline CAMPEP sequences & fit to T2-A1 in the anterior \\
\hline T2-A1 & DPLDRLLSTLTDLEDRYVAEQKEDDAKNQEYQGACTVDISAFDKDLAESNRKKIELEARLEGQLYPQRSILEGLVAQKKAEVKGYQKDLDELDAQ \\
\hline CAMPEP 0190380732 & NPIAKVLELLEGMRGKIVAEGEEEQATYEEYTGWCRKESKALKREVADAQAQVKELTATIEKÃTATĨDE-LTEKTSDLAASITSAQNNDLKDATAV \\
\hline CAMPEP_0190304660 & SPISKVIQLLSSLESKVTSEGEKEQQQYEEFARWCEENAKEKQHELKNSAERKESLESTLEKTQADIST-TKAKIEELSSS ISQNDADLEKATAV \\
\hline CAMPEP_0190331188 & NPVSRVVEVLTSMKAEVEQQGQTDEEHYQKFSCWCTTNKQSKQNAISRAEDQSTQLQATVLENVATKSQ-LETEIGQLKKARATAKKSLDTATSL \\
\hline CAMPEP_0190324578 & NPVRKVVDLLQKLGKEIEDETSKAKKMYNKYMCYCDTNTÁSLEKDIEEEKEH ISELEANMKELSGQNAQ-LEAEIADAQESLEANKKTIAEATVM \\
\hline continued & 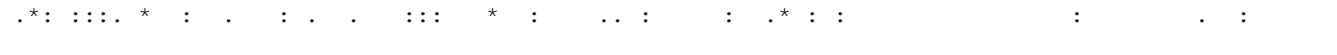 \\
\hline $\mathrm{T} 2-\mathrm{A} 1$ & RAEEHEDFEEKVLEHQEATAIIAEARRLFADNIEHGS-FIQ \\
\hline CAMPEP_0190380732 & REKEHQDYAAVAAELQEAIDTIERGIAVLEREMPKGS-FMQ \\
\hline CAMPEP- 0190304660 & RKAEHKDFTERDQESVETIDTLERALQTIKKELAHGF-VQ- \\
\hline CAMPEP-0190331188 & RTREHQDFETIQSDLKTAIGQLQRAVKIVESNFGSGDSFLQ \\
\hline CAMPEP_0190324578 & RNNEAAQYASESGELKDSIAALAKAIPALKKGMENGS-FL- \\
\hline $\begin{array}{l}\text { CAMPEP sequence wl } \\
\text { T2-A1 }\end{array}$ & $\begin{array}{l}* \quad * \quad:: \quad: \quad: \quad: \ldots \quad . \quad: \quad * \quad . \\
\text { fits to T2-A1 in the posterior } \\
\text { DPLDRLLSTLTDLEDRYVAEQKEDD-- }\end{array}$ \\
\hline $\begin{array}{l}\text { CAMPEP_0190315454 } \\
\text { Continued } \\
\text { T2-A1 }\end{array}$ & $\begin{array}{l}\text { HPLTKIVNLLKDMEAQLTADAKADKTTYEKLACWCKNTKESKVAAISTAQSRITDLTAAVQEYAASEAEADTNIKQLKKEVNQGENALAQARAGR } \\
\cdot{ }^{* *}::{ }^{*} \cdot{ }^{*}:{ }^{*}: * *\end{array}$ \\
\hline $\begin{array}{l}\text { continued } \\
\text { T2-A1 }\end{array}$ & EQAAAAWYADEKDLVQA IAALKNAIQQLSLRGGASL I H IGHVAERVMTRHEANFVGKMTQEQRQVIAALQQPASTGSYTLQS DKIMGVLSSMLEE \\
\hline $\begin{array}{l}\text { CAMPEP_0190315454 } \\
\text { Continued } \\
\text { T2-A1 }\end{array}$ & MEKDLATGRSDDAMAASQYENLKADKTAENAAGKQAIRTTKTEASAAATNLERSKEDLSDTRAALDADRKFLSDLNLRCQNVDHDYSERVSDRSA \\
\hline CAMPEP_0190315454 & EISAVQDAITILTEDSGSETMGRALSLLQVGAARAAALSREARGRVAGLLLAAAKRTGNADLLKLAGTVSLDTFTKIKAACEKMISELKQQQVDE \\
\hline continued & \\
\hline T2-A1 & QEYQGACTVDISAFDKDLAESNRKKIELEARLEGQLYPQRSILEGLVAQKKAEVKGYQKDLDEL-------DAQRAEEHEDFEEKVLEHQEATAI \\
\hline $\begin{array}{l}\text { CAMPEP_0190315454 } \\
\text { continued }\end{array}$ & 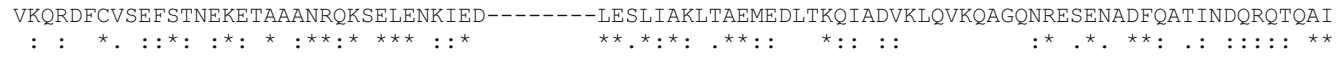 \\
\hline T2-A1 & IAEARR----LFAD--------NIEHGSFIQ \\
\hline CAMPEP_0190315454 & $\begin{array}{l}\text { LAKAITRLQVVYAKTQEERLAASGSAGALVQ } \\
:^{\star}:{ }^{\star} \quad::^{\star} \\
\end{array}$ \\
\hline T2-A2 & LADAGAVQKIIDLADELLAKI - - - - \\
\hline $\begin{array}{l}\text { CAMPEP_0190318812 } \\
\text { Continued }\end{array}$ & $\begin{array}{l}\text { AVQVTPLQKVVEMLEGMLAKGKEEKHDEVVRFNTFKQWCDDTNNAKRATVKDEKHSINKLTANIEKDTATIAHLDDELARLNDDLAAWQGDLKAA } \\
.: \quad: * *::: \quad: \quad * * * \\
\text { * * * }\end{array}$ \\
\hline $\begin{array}{l}\text { CAMPEP_0190318812 } \\
\text { continued }\end{array}$ & $\begin{array}{l}\text { AGIRKKDNIDFMEQHKDYTTS IDQLQQAVES IKSHSHDAPQSSALLQLQNNKLIPTSAYRVLQAFLQTSDDGVPEANAYESQSGGVVEVLTELRR } \\
\qquad{ }^{* *}::\end{array}$ \\
\hline T2-A2 & -AEDKRVE----------AYKKSRNF IVISLNVAGSALANATSDLASLNDI IAQVEASLDTTEQRIENVSADRHDRFTQCEEAVQDYQDARAART \\
\hline CAMPEP_0190318812 & KFEDERLQLEKEEANAKFAYQQAQQGLSDNIEQAETELATKTQDMQKTKKRKAÄDEEELETTQKALAEDSAYLQDTENLCLEKAQAYENRQVTRA \\
\hline continued & 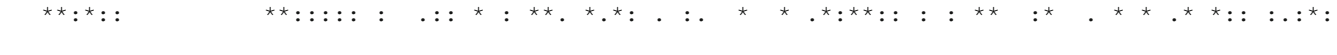 \\
\hline T2-A2 & SDRDVVSQTIGLVNKELRTLREQ---------LALRQQAGEEI \\
\hline CAMPEP_0190318812 & 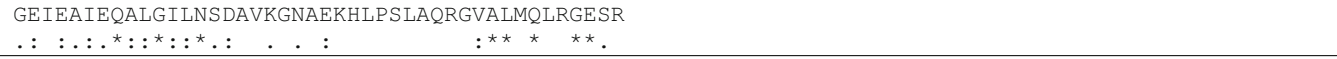 \\
\hline T2-B1 & DPLDRLVSTLTDLEDRYVAEQKEDDAKNQEYQGACTVDITAFDKDLAESNRKKIELEARLEGQLYPQRSILEGLVAQKKAEVKGYQKDLDELDAQ \\
\hline CAMPEP_0190331188 & NPVSRVVEVLTSMKAEVEQQGQTDEEHYQKFSCWCTTNKQSKQNAISRAEDQSTQLQATVLENVATKS-QLETEIGQLKKARATAKKSLDTATSL \\
\hline CAMPEP_0190380732 & NPIAKVLELLEGMRGKIVAEGEEEQATYEEYTGWCRKESKALKREVADAQAQVKELTATIEKATATIDELTE-KTSDLAASITSAQNDLKDATAV \\
\hline continued & 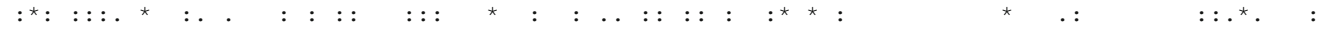 \\
\hline T2-B1 & RAEEHEDFEEKVLEHQEATAIIAEARRLFADNIEHE-SFVQ \\
\hline CAMPEP 0190331188 & RTREHQDFETIQSDLKTAIGQLQRAVKIVESNFGSGDSFLQ \\
\hline CAMPEP_0190380732 & REKEHQDYAAVAAELQEAIDTIERGIAVLEREMPKG-SFMQ \\
\hline & 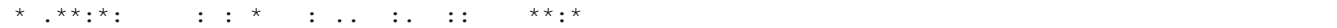 \\
\hline
\end{tabular}


Table 1 (continued)

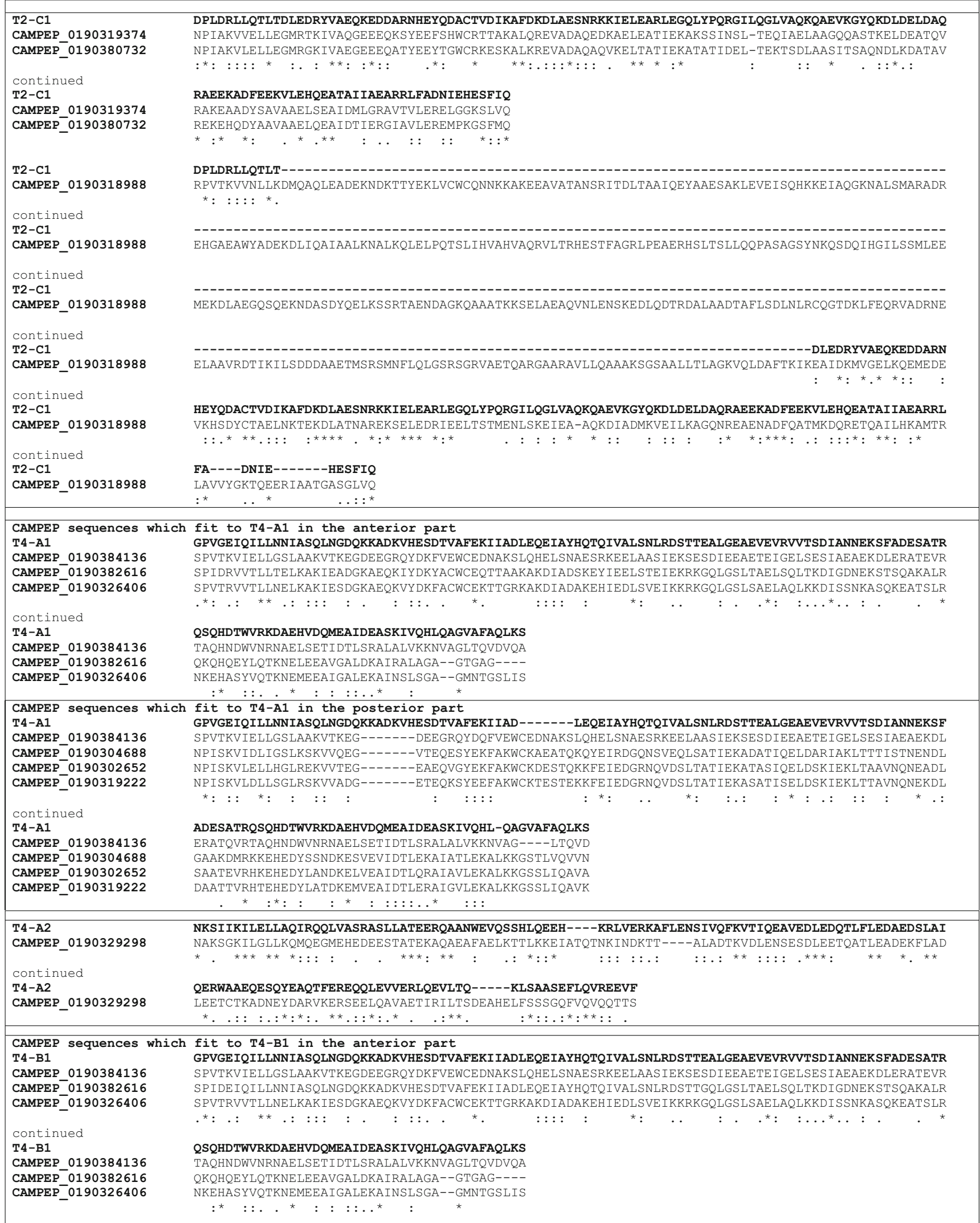


Table 1 (continued)

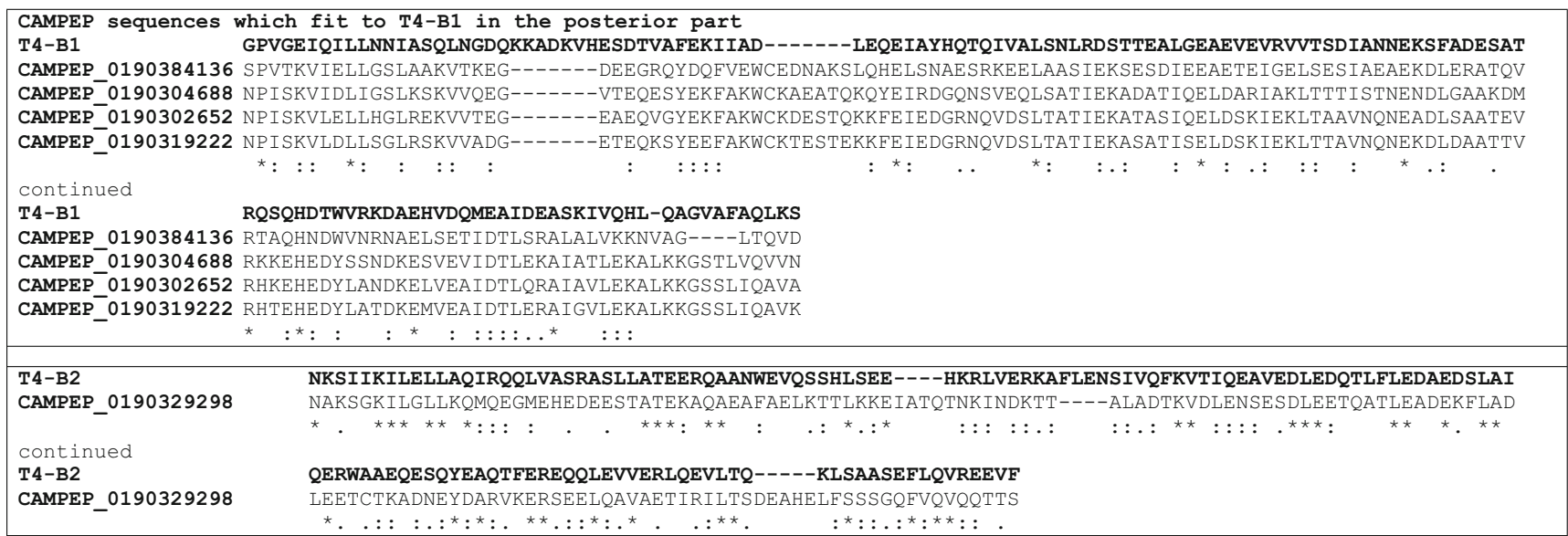

